\title{
Mineralogía y paragénesis de dachiardita cálcica en rocas volcánicas cretácicas ceolitizadas, Esquel, Chubut, Patagonia argentina
}

María E. Vattuone

Carlos O. Latorre

Pablo R. Leal
Facultad de Ciencias Exactas y Naturales, Universidad de Buenos Aires, Ciudad Universitaria CP 1428EHA, Buenos Aires, Argentina-INGEIS, CONICET, Ciudad Universitaria, CP 1428EHA, Buenos Aires, Argentina elena@gl.fcen.uba.ar

INGEIS, CONICET, Ciudad Universitaria, CP 1428, Buenos Aires, Argentina latorre@gl.fcen.uba.ar

Facultad de Ciencias Exactas y Naturales, Universidad de Buenos Aires, Ciudad Universitaria, CP 1428EHA, Buenos Aires, Argentina leal@gl.fcen.uba.ar

RESUMEN

Se halló dachiardita cálcica en brechas de rocas volcánicas cretácicas afectadas por metamorfismo de muy bajo grado. El cemento de la brecha presenta cuarzo, dachiardita cálcica, mordenita, cristobalita, calcita y siderita, mientras que en los clastos hay pectolita, prehnita, yugawaralita, dachiardita cálcica, albita, adularia, interestratificados de esmectita clorita (S/C), cuarzo y cristobalita. La mayor parte de dachiardita se halla en el cemento de la brecha, donde forma estructuras tubulares que contienen cristales hojosos a tabulares, paralelos o levemente radiados dispuestos en capas concéntricas y elongados a lo largo del eje cristalográfico 'b' y de los tubos; los cristales están achatados según $\{001\}$ o raramente $\{100\}$. Muestra maclas cíclicas en la sección perpendicular al alargamiento y maclas múltiples paralelas a $\{001\}$, aunque también hay individuos sin maclar. Las características ópticas son: $\alpha=b ; \gamma: c=43^{\circ} ; 2 \mathrm{~V} \alpha=55^{\circ}$ / $60^{\circ}$; dispersión fuerte $r>v$. La fórmula estructural es: $\mathrm{Ca}_{1,54-1.72} \mathrm{~K}_{0,82-0,98} \mathrm{Na}_{0}\left(\mathrm{Al}_{3,65-3,98} \mathrm{Si}_{19,95-20,25} \mathrm{O}_{48}\right) 13 \mathrm{H}_{2} \mathrm{O} ; \mathrm{T}_{\mathrm{Si}}=0,83-0,85$; $R^{2+} /\left(R^{+}+R^{2+}\right)=0,61 / 0,68 ; D E C=$ Ca y K. Las líneas de difracción más fuertes son: $3,460 \AA ; 3,190 \AA$ y $1,870 \AA ; a_{0}=18,679$ $\AA ; b_{0}=7,488 \AA ; c_{0}=10,267 \AA ; \beta=107^{\circ} 86^{\prime}$. La sucesión mineralógica secundaria depositada con el descenso de la temperatura y cambios en la $\mathrm{X}_{\mathrm{co} 2}$ sería: calcita (etapa I); pectolita, prehnita, yugawaralita, cuarzo, dachiardita cálcica, interestratificados S/C, adularia, albita (etapa II, facies de ceolita); mordenita y cristobalita (etapa III) y por último, calcita y siderita (etapa IV). Las paragénesis metamórficas indican temperaturas de formación para la dachiardita cálcica de 200 a $220^{\circ} \mathrm{C}$ a presiones menores que $0,5 \mathrm{~kb}$, a partir de fluidos enriquecidos en $\mathrm{a}_{\mathrm{Si}}$ y $\mathrm{a}_{\mathrm{Ca}}$.

Palabras claves: Dachiardita cálcica, Metamorfismo de muy bajo grado, Cretácico, Esquel, Patagonia argentina.

\section{ABSTRACT}

Mineralogy and paragenesis of Ca-dachiardite in Cretaceous zeolitized volcanic rocks, Esquel, Chubut, Argentinian Patagonia. Ca-dachiardite is present in Cretaceous volcanic breccias affected by very low grade metamorphism. The cement of the breccia shows quartz, Ca-dachiardite, mordenite, cristobalite, calcite and siderite, while in the clasts there are pectolite, prehnite, yugawaralite, Ca-dachiardite, albite, adularia, interestratified smectite/ chlorite $(\mathrm{S} / \mathrm{C})$, quartz and cristobalite. Most Ca-dachiardites are found in the cement of the breccia. It occurs principally as fibrous tubes of parallel or lightly divergent bladed and tabular crystals, arranged in concentrical layers with length 
along ' $b$ ' and the fibers; the crystals are flattened on $\{001\}$ and rarely on $\{100\}$. It shows cyclic twins in cross section normal to the length and multiple twins parallel to $\{001\}$, but single crystals are also present. The optical character shows: $\alpha=b$; $\gamma: \mathrm{C}=43^{\circ} ; 2 \mathrm{~V} \alpha=55^{\circ} / 60^{\circ}$; strong dispersion $\mathrm{r}>\mathrm{v}$. The structural formulae is: $\mathrm{Ca}_{1.54-1.72} \mathrm{~K}_{0.82-0.98} \mathrm{Na}_{0}\left(\mathrm{Al}_{3.65-3.98} \mathrm{Si}_{19.95-20.25} \mathrm{O}_{48}\right)$ $13 \mathrm{H}_{2} \mathrm{O} ; \mathrm{T}_{\mathrm{Si}}=0.83-0.85 ; \mathrm{R}^{2+} /\left(\mathrm{R}^{+}+\mathrm{R}^{2+}\right)=0.61 / 0.68 ; \mathrm{DEC}=\mathrm{Ca}$ and $\mathrm{K}$. The strong diffraction lines are: $3.460 \AA ; 3.190 \AA$ and $1.870 \AA ; a_{0}=18.679 \AA ; b_{0}=7.488 \AA ; c_{0} 10.267 \AA ; \beta=107^{\circ} 86^{\prime}$. The secondary mineralogical succession deposited according with the decrease in temperature and $\mathrm{X}_{\mathrm{co} 2}$, could be: calcite (step I); pectolite, prehnite, quartz, yugawaralite, Cadachiardite, S/C, adularia, albite (step II, zeolite facies); mordenite and cristobalite (step III) and then, siderite and calcite (step IV). The metamorphic assemblages suggests that the crystallization conditions of Ca-dachiardite would be 200$220^{\circ} \mathrm{C}$ at pressures smaller than $0.5 \mathrm{~kb}$, from high $a_{\mathrm{Si}}$ and $a_{\mathrm{Ca}}$ fluids.

Key words: Ca dachiardite, Very low grade metamorphism, Cretaceous, Esquel, Patagonia argentina.

\section{INTRODUCCIÓN}

La Cordillera Patagónica Septentrional desarrolló un margen activo en forma casi continua desde el Jurásico hasta la actualidad. La evolución de los consecuentes arcos no sólo generó un extenso magmatismo sino también importantes procesos de metamorfismo de muy bajo grado (MMBG) e hidrotermales. Entre los minerales secundarios generados allí por estos procesos son frecuentes las ceolitas que se encuentran fundamentalmente en coladas de basaltos y/o andesitas.

Las ceolitas son silicatos con estructura tridimensional y abierta, y con moléculas de agua en sus estructuras. Su especial disposición cristalográfica (tamices moleculares) permite una gran variedad de aplicaciones, por lo que se consideran minerales de amplia aplicación en el futuro Esa estructura, provista de canales difiere según las especies y determina el CEC (capacidad de intercambio catiónico) que favorece o inhibe la penetración de fluídos. Por ser un grupo sistemático no bien conocido aún, existen especies con estructuras complejas y composición quimica variable, cuyo hallazgo es novedoso en mineralogía. Este es el caso de la dachiardita cálcica que es encontrada por primera vez en América del Sur.

La abundancia de minerales del grupo de las ceolitas convierte al sector de los Andes Patagónicos Septentrionales en un entorno capaz de ofrecer una vasta variedad de especies que permiten estudiar la sistemática del grupo, sus asociaciones minerales y texturas, para conocer no sólo las características mineralógicas sino también sus condiciones de formación. Prueba de ello, son las numerosas especies ya descritas en esa área por los autores de este trabajo y colaboradores (Depine et al., 2003; Lagorio et al., 2001; Latorre et al., 1990 y 2000; Tourn y Vattuone, 2002; Vattuone y Latorre, 1990, 1996a y b, 1999 y 2002; Vattuone et al., 1996, 1999 y 2001 a y b.)

En esta oportunidad se describe la mineralogía de dachiardita cálcica, una ceolita encontrada constituyendo el cemento de brechas volcánicas cretácicas afectadas por metamorfismo de muy bajo grado, al este del cerro Nahuel Pan, en Esquel, Chubut (Fig. 1). Los objetivos del trabajo son: el estudio mineralógico de la dachiardita, la determinación de su génesis y sus asociaciones.

La dachiardita es un tectosilicato correspondiente al grupo de las ceolitas relacionadas con mordenita según la clasificación de Dana's New Mineralogy (Gaines et al., 1997); su estructura (Tipo DAC) es peculiar y compleja con desorden y maclado que origina en la mayor parte de los casos difusos 'peaks' en la Difracción de Rayos X (DRX). Cristaliza en el sistema monoclínico, grupo puntual $2 / m$ y grupo espacial que resulta en una simetría promedio C2/m (Armbruster y Gunter, 2001).

Es una ceolita rica en sílice, muy poco frecuente, que se conoce solamente en una docena de localidades en distintas partes del mundo. La variedad cálcica se menciona en la localidad de San Piero, Elba, Italia (Bermann, 1925, Gottardi y Galli, 1985); en Yellowstone National Park, Wyoming, USA (Bargar y Beeson, 1984 y Bargar et al., 1987); en Onoyama Mine, Japan y en Ogasawara Islands, Japan (Nishido y Otsuka, 1981).

La serie dachiardita, comprende una variedad cálcica y otra sódica (Coombs, 1997). Anteriormente, el nombre dachiardita s/l se usaba sólo para la variedad cálcica y a la variedad sódica se la llamaba dachiardita sódica (Gaines et al., 1997). Aunque presenta $\mathrm{K}$ como catión intercambiable, 
todavía no ha sido definida una variedad potásica, la cual también podría formar parte de la serie.

La localidad tipo es San Piero in Campo, Elba (Italia), en donde la dachiardita cristaliza como individuos maclados con maclas cíclicas de ocho individuos ('eightling'), en una pegmatita granítica (Bermann, 1925; Gottardi y Galli,1985).

La dachiardita se presenta en la mayoría de los casos como fibras, cristales prismáticos finos o agregados radiado divergentes o paralelos de hojas o fibras (Yoshimura y Wacabayasi, 1977; Nishido y Otsuka, 1981). Bargar et al. (1987) describieron cristales prismáticos achatados según $\{001\}$ y menos comúnmente $\{100\}$. Son comunes las maclas irregulares y complejas y fallas de apilamiento que afectan a la estructura y que indican desorden (Gellens et al., 1982; Armbuster y Gunter, 2001; Gaines et al., 1997).

\section{CONTEXTOGEOLÓGICO}

Los afloramientos portadores de dachiardita corresponden a una secuencia volcánica de composición intermedia ubicada al este del Cerro Nahuel Pan (Fig. 1).

Según los trabajos de Lizuaín (1999), entre otros, en el Cerro Nahuel Pan predominan las rocas volcánicas del Jurásico Inferior sobre las cuales se apoyan las asociaciones volcaniclásticas del Jurásico Medio y Superior. Las secuencias situadas al este del Cerro Nahuel Pan, donde se encuentra el área de este trabajo (Fig. 1), son consideradas según el Mapa Geológico de Chubut
(Secretaría de Minería, 1995) como pertenecientes al Grupo Divisadero de edad cretácica; Vattuone y Latorre (2004) han confirmado esa edad en un miembro de la secuencia por dataciones radimétricas. Afloramientos que se consideraron correlacionables con el Grupo Divisadero, fueron estudiadas en Chubut (Ramos,1976, 1978 y 1981; lanizzoto et al., 2003 y Folguera e lanizzotto, 2004) en el Lago La Plata; en la región cordillerana entre los $43^{\circ}$ y $47^{\circ}$ LS en el sector argentino (Haller y Lapido,1982 y Pesce, 1978) y en el sector chileno (De la Cruz et al., 2003). Trabajos regionales de

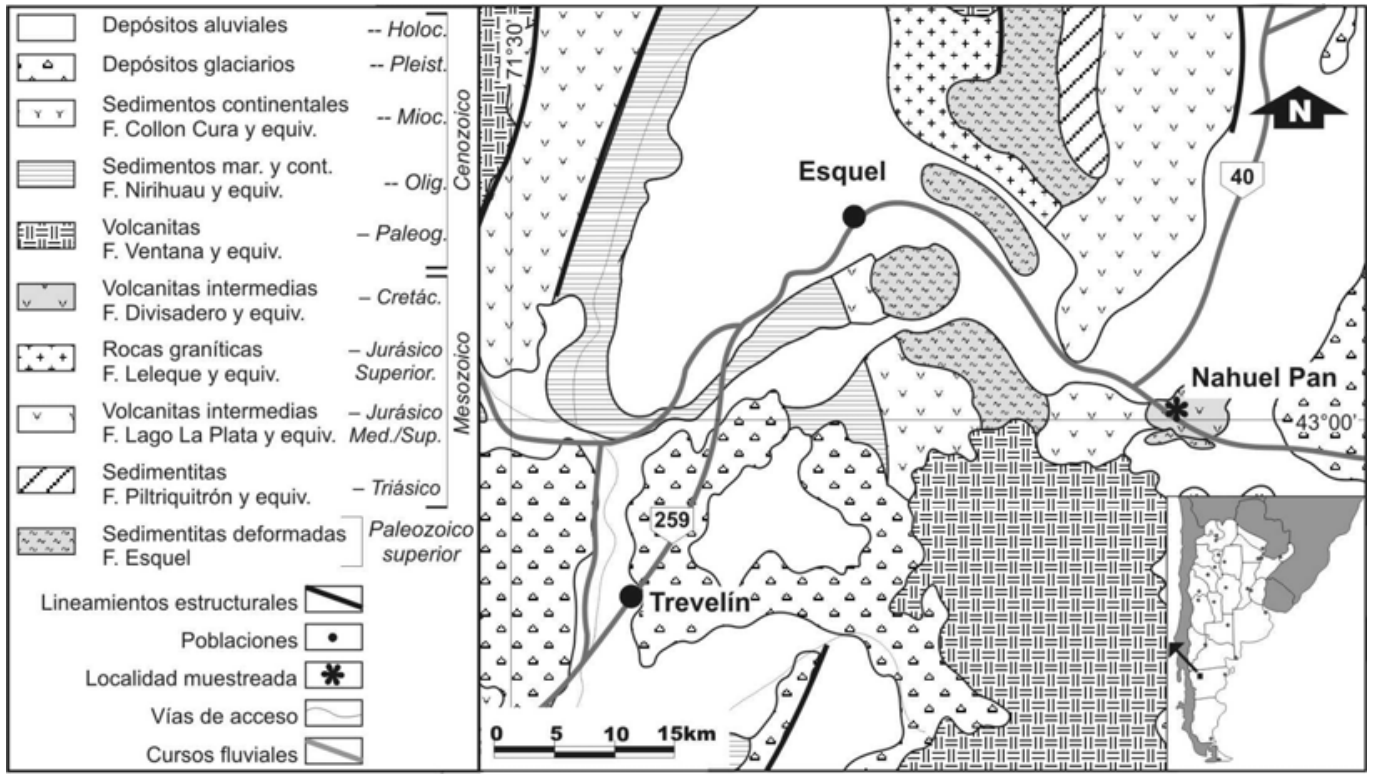

FIG. 1. Mapa geológico y de ubicación del área indicando la localidad muestreada (modificado sobre la base del Mapa Geológico de Chubut (Secretaría de Minería, 1995) y Lizuaín (1999). 
estratigrafía, petrología y geocronología sobre las volcanitas cretácicas de Chubut, han sido realizados, además, por Pankhurst et al. (1998), entre otros.

\section{DESCRIPCIÓN DE LOS AFLORAMIENTOS}

Los mantos lávicos de composición dacítico/ andesítica del Grupo Divisadero que se extienden en la base del Cerro Nahuel Pan son de colores verdosos en fractura fresca y con pátina ocre cuando están alterados. Tienen de 2 a 3 metros de espesor y se presentan flanqueados por bancos de ignimbritas de color gris con fragmentos líticos y "fiammes'. Ese conjunto está cubierto, a su vez, por mantos lávicos que presentan brechamiento de origen mecánico, con clastos de color verde con pátina rojiza, que fueron afectados por alteración hidrotermal que produjo la formación de minerales secundarios de metamorfismo de muy bajo grado. El cemento de la brecha está constituido mayoritariamente por venas portadoras de carbonatos, minerales de sílice, dachiardita y mordenita.

Toda la secuencia lávico-piroclástica es homoclinal y tiene rumbo noreste siguiendo fracturas regionales e inclinando $10^{\circ}$ al noroeste. Los mantos lávicos dacítico-andesíticos frescos fueron datados por el método de K/Ar sobre roca total, en $105 \pm 5 \mathrm{Ma}$ (Vattuone y Latorre, 2004).

Esos mantos (Fig. 1), se apoyan en discordancia angular sobre rocas volcánicas más antiguas, de composición basáltico-andesítica, las que en el sector estudiado fueron datadas en $252 \pm 10$ Ma por $\mathrm{K} / \mathrm{Ar}$ sobre roca total (Vattuone y Latorre, 2004); las mismas no fueron representadas en la figura 1 porque no lo permitio su escala. Esa edad es novedosa para el área, ya que en general, la Formación Divisadero suele suprayacer a rocas volcánicas que se atribuyen a la Formación Lago La Plata de edad jurásica. El perfil de la secuencia y un mayor detalle de los análisis geocronológicos se pueden encontrar en Vattuone y Latorre (2004).

\section{PETROGRAFÍA DE LAS BRECHAS VOLCÁNICAS}

Los clastos de la brecha están conformados por dacitas y andesitas porfíricas con abundantes fenocristales hipidiomorfos a idiomorfos de andesina seriados, con macla múltiple y de Carlsbad o con zonación, agrupados en glomérulos, casi totalmente alterados a ceolitas, prehnita, calcita y escasa albita. Hay algunos fenocristales de anfíbol bordeados por magnetita y reemplazados parcialmente por interestratificados no regulares de esmectita/clorita (S/C). La matriz es microgranular, aunque suele tener en sectores cierta fluidez y está integrada por microlitos de plagioclasa con diverso grado de alteración, S/C, pequeños cristalitos de pumpellyita que a veces conservan la característica forma en 'hoja de arce' y están reemplazados por S/ C y microfenoblastos de adularia y ceolitas. Se observan venas rellenas con S/C y prehnita que atraviesan a los clastos.

El cemento de la brecha está compuesto fundamentalmente por carbonatos (calcita y siderita), variedades de sílice (cuarzo y cristobalita), dachiardita y mordenita. Se observó la presencia de albita en un sector de las rocas.

\section{ASOCIACIONES MINERALES METAMÓRFICAS}

Los minerales secundarios se encuentran en distintos microdominios: como reemplazo total o parcial de minerales primarios relictos de los clastos de la brecha en fenocristales y en la matriz, o como cemento de la brecha.

Fenocristales: reemplazos parciales o totales de las fases ígneas primarias (plagioclasa y anfíbola) por: yugawaralita, prehnita, albita, S/C, magnetita y hematita; en menor proporción por calcita en'pequeñas islas' en las plagioclasas.

Matriz: reemplazos parciales de la matriz por adularia, escasa albita, S/C, pectolita, escasa pumpellyita alterada a $\mathrm{S} / \mathrm{C}$, yugawaralita, dachiardita calcica, cuarzo y calcita.

Cemento y venas: el cemento de la brecha está constituido por carbonatos con mordenita y cristobalita de baja temperatura y en otros sectores por cuarzo, dachiardita calcica y S/C con calcita de segunda generación alterando a la dachiardita. Hay venas de algunos milímetros de ancho con S/ $C$ y cristales fibrosos y radiales de prehnita con minúsculos cristalitos de pectolita incluídos. Otras presentan una estructura zonal con borde de calcita, seguida por dachiardita calcica, cuarzo, a veces nuevamente dachiardita, cuarzo y albita. 


\section{MINERALOGÍA DE DACHIARDITA}

\section{CARACTERÍSTICAS FÍSICAS Y ÓPTICAS}

La dachiardita de Esquel se presenta comúnmente formando estructuras tubulares a veces incompletas de diámetro variable hasta de $0,2 \mathrm{~mm}$ (Fig. 2A). Esas estructuras están rellenas por cristales hojosos a tabulares delgados, dispuestos en forma paralela a la longitud de los tubos o levemente radiado divergente (Fig. 2B) y en capas concéntricas alrededor de otros cristales de dachiardita o de cristales de cuarzo. Las diferentes capas de dachiardita presentan discontinuidades producto de la variación en la orientación óptica de los cristales en cada una de ellas. Excepcionalmente los cristales pueden tener hábito prismático-tabular.

En algunos casos, el depósito de dachiardita se alterna rítmicamente con el de $\mathrm{S} / \mathrm{C}$ lo que constituye bandas concéntricas de agregados hojosos radiadodivergentes de dachiardita con muy delgadas bandas de S/C configurando textura en escarapela.

La ceolita es traslúcida e incolora y con brillo sedoso en las secciones con clivaje y rosada, con brillo graso en las secciones perpendiculares a la elongación de las fibras.

Los cristales son elongados según el eje cristalográfico 'b' y tienen clivajes perfectos paralelos a $\{100\}$ y $\{001\}$ (Fig. 2C). Son achatados comúnmente según 'c' y menos comúnmente según 'a'. Se observaron las formas $\{001\},\{100\},\{010\}$, $\{$ hk0\}, $\{0 \mathrm{kl}\}$ y $\{\mathrm{hkl}\}$ (Figs. 2D y $2 \mathrm{E}$ ).

Las secciones perpendiculares a las estructuras tubulares muestran, a veces, cambio en la orientación óptica por sectores, que parecen constituir maclas cíclicas, similares a las descritas por Berman (1925) y Gottardi y Galli (1985), para la dachiardita de Elba.

En [001] se observan maclas periódicas múltiples paralelas a $\{001\}$. Sobre $\{010\}$, se ve la traza de estas maclas y las fallas de apilamiento perpendiculares (Fig. 2F). Esto se asemeja a lo esquematizado por Gellens et al. (1982) (Fig. 3).

Además de individuos maclados de dachiardita se han observado individuos simples, sin maclas. A veces existe textura en 'hourglass', lo que fue mencionado también por Bonardi et al. (1981) para la dachiardita $\mathrm{Na}$ de Montreal, Canadá.

La relación entre la simetría óptica y cristalo- gráfica indica que $\alpha$ coincide con b y con el alargamiento de los cristales y que el ángulo $\gamma$ :c es de $43^{\circ}$. Esto está de acuerdo con lo expresado por Bargar y Beeson (1984) y Bargar et al. (1987) para la dachiardita de Yellowstone y por Gaines et al. (1997) y coincide con la orientación indicada por Deer et al. (2004) (Tabla 1).

Las figuras de interferencia obtenidas en caras ligeramente oblicuas a $\{010\}$ presentan la bisectriz aguda asimétrica, con un $2 \mathrm{Va}=55 / 60^{\circ}$ y en caras \{hk0\} se obtiene la figura de inteferencia perpendicular a un eje óptico. La dispersión es fuerte $(r>v)$. El valor del $2 \mathrm{~V}$ y dispersión están dentro de los normales para dachiarditas según Deer et al. (2004).

\section{QUÍMICA MINERAL}

Los análisis químicos por EDS (dominant exchange cations) (Tabla 2) se efectuaron sobre muestras pulidas de dachiardita del cemento de la brecha. De acuerdo a los datos obtenidos (a.p.f.u./ $\mathrm{O}_{48}$ los valores de Si del mineral estudiado en este trabajo oscilan entre 19,95 y 20,25 ; los de Al entre 3,65 y 3,98 ; los de $\mathrm{K}$ entre 0,82 y 0,98 y los de $\mathrm{Ca}$ entre 1,54 y 1,72 .

La ceolita de Esquel, tiene la siguiente fórmula: $\mathrm{Ca}_{1,54-1,72} \mathrm{~K}_{0,82-0,98} \mathrm{Na}_{0}\left(\mathrm{Al}_{3,65-3,98} \mathrm{Si}_{19,95-20,25} \mathrm{O}_{48}\right) 13 \mathrm{H}_{2} \mathrm{O}$. La composición química de la dachiardita difiere según las localidades en el mundo, ya que es ampliamente variable no sólo en los cationes intercambiables sino en la relación Si/Al. En la localidad tipo (Elba, Italia; Gottardi y Galli, 1985) según Vezzalini (1984) tiene una fórmula de: $\left(\mathrm{Ca}_{1,54} \mathrm{~K}_{0,92}\right.$ $\left.\mathrm{Na}_{0,42} \mathrm{Cs}_{0,11} \mathrm{Sr}_{0,12} \mathrm{Ba}_{0,01}\right)\left(\mathrm{Al}_{4,86} \mathrm{Si}_{18,96} \mathrm{O}_{48}\right) 12,56 \mathrm{H}_{2} \mathrm{O}$, la cual es diferente a la mayor parte de las dachiarditas en cuanto a la presencia de $\mathrm{Cs}$ y $\mathrm{Sr}$ que en general este mineral no posee. En cuanto a las proporciones relativas de los DEC ('dominant exchange cations') $=\mathrm{Ca}>\mathrm{K}>\mathrm{Na}$, los especímenes de Esquel se asemejan a esta dachiardita, a los de Onoyama Mine (Nishido y Otsuka, 1981) y a algunos mencionados por Bargar et al. (1987) para Yellowstone.

La relación $\mathrm{T}_{\mathrm{Si}}=0,83-0,85$ es normal para la especie ya que los valores oscilan entre $0,77 \mathrm{y}$ 0,86 . La relación entre cationes divalentes y mono- 


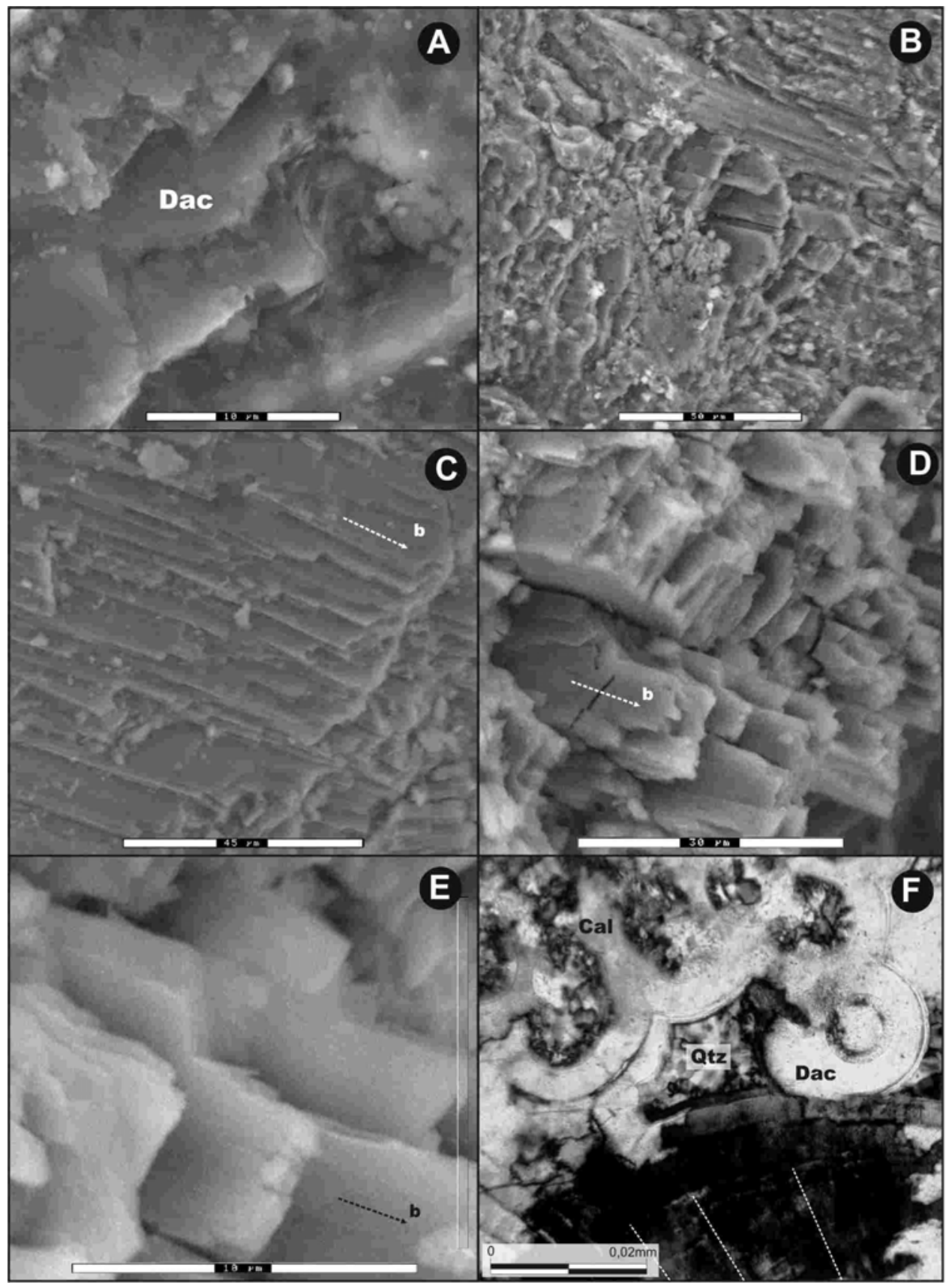

FIG. 2. Microfotografías: A- estructura tubular abierta de dachiardita (Dac); B-cristales de dachiardita que en el centro de la foto muestran una estructura tubular integrada por cristales delgados, dispuestos paralelamente al alargamiento del tubo; C- cristales hojosos de dachiardita, elongados según el eje 'b' que muestran los dos clivajes perfectos paralelos a la elongación; D- cristales hojosos de dachiardita elongados según 'b' y achatados según 'c' donde se distinguen $\{001\},\{100\}$ y $\{\mathrm{hk} 0\}$; E- cristales tabulares de dachiardita elongados según 'b' donde se observan $\{001\}\{100\}$ y $\{010\}$; F-vena de dachiardita (Dac), cortada perpendicularmente a las estructuras tubulares; en la parte inferior de la fotografía se encuentran estructuras concéntricas que en el borde externo, muestran cristales hojosos achatados según $\{001\}$ y hacia la parte interna, cristales cortados según $\{010\}$ donde se observan las trazas de las maclas múltiples paralelas a $\{001\}$ (líneas punteadas) y fallas de apilamiento perpendiculares. Entre la dachiardita se observa cuarzo (Qtz); la calcita (Cal), reemplaza en algunos sectores a las estructuras tubulares. A,B,C,D y E, fueron tomadas con microscopio electrónico y $\mathrm{F}$ con microscopio de polarización y polarizadores cruzados. 


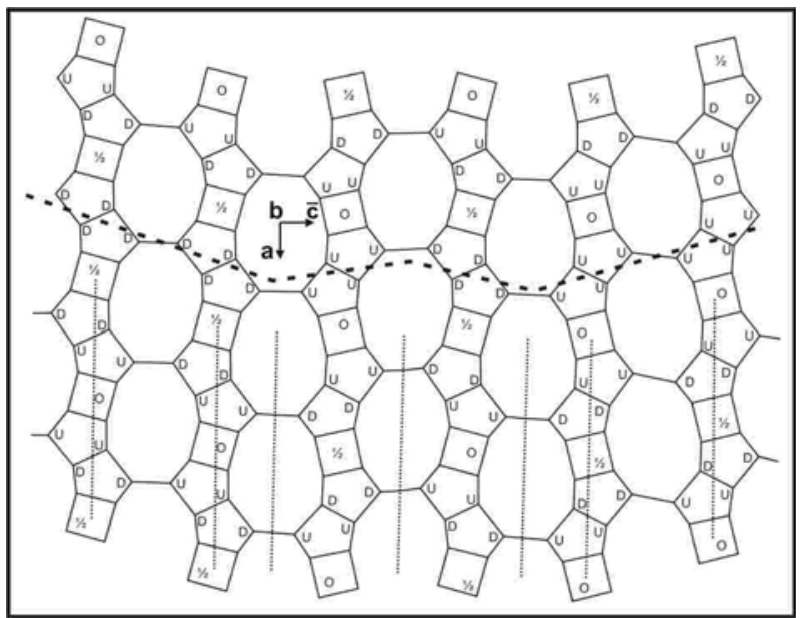

FIG. 3. Esquema de Gellens et al. (1952), aplicable a la dachiardita de este trabajo, que muestra el maclado múltiple \{001\} y las fallas de apilamiento en la sección perpendicular a 'b' cristalográfico. La línea punteada gruesa marca las fallas de apilamiento y las líneas punteadas en la dirección de 'a' cristalográfico, los planos de macla. 1/2 es la unión de dos tetraedros colocados hacia abajo (D) y 0 la de tetraedros apuntando hacia arriba (U).

TABLA 1. CUADRO COMPARATIVO CON LAS CARACTERÍSTICAS MINERALÓGICAS DE LA DACHIARDITA DE ESQUEL Y LAS PUBLICADAS POR OTROS INVESTIGADORES.

\begin{tabular}{|c|c|c|}
\hline Dachiardita Esquel & \multicolumn{2}{|c|}{ Otras dachiarditas } \\
\hline$a_{0}=18,679 \AA$ & $a_{0}=18,625-18,730 \AA$ & \multirow{4}{*}{ Armbrustery Gunter (2001) } \\
\hline $\mathrm{b}_{0}=7,488 \AA$ & $b_{0}=7,489-7,540 \AA$ & \\
\hline $\mathrm{C}_{0}=10,267 \AA$ & $c_{0}=10,239-10,310 \AA$ & \\
\hline$\beta=107^{\circ} 86^{\prime}$ & $\beta=107^{\circ} 87-108^{\circ} 74$ & \\
\hline $\mathrm{b} \equiv \alpha ; \gamma^{\wedge} \mathrm{c}: 43^{\circ}$ & $\mathrm{b} \equiv \alpha ; \gamma^{\wedge} \mathrm{c}: 18 / 58^{\circ}$ & Deer et al. (2004) \\
\hline $\begin{array}{l}\text { Hábito tabular; laminar } \\
\text { Achatado según }\{001\} \text { y -común } \\
\{100\} . \text { Elongado según b }\end{array}$ & \begin{tabular}{|lll} 
Tabular, laminar \\
Achatado según $\quad\{001\} \quad$ y \\
elongado según b
\end{tabular} & $\begin{array}{l}\text { Deer et al. (2004) } \\
\text { Bargar et al. (1987) }\end{array}$ \\
\hline Clivaje perf; $\{100\}$ y $\{001\}$ & Clivaje perfil; $\{100\}$ y $\{001\}$ & Deer et al. (2004) \\
\hline $\begin{array}{l}\text { Formas comunes: }\{001\},\{100\} \\
\{010\} ;\{\mathrm{hk} 0\} ;\{0 \mathrm{kl}\} \text { y }\{\mathrm{hkl}\}\end{array}$ & $\begin{array}{l}\text { Formas comunes: }\{001\}, \\
\{100\},\{110\}\end{array}$ & Deer et al. (2004) \\
\hline $\begin{array}{l}\text { Maclas: múltiples paralelas a } \\
\{001\} \text { y fallas de apilamiento } \\
\{100\} ; \text { maclas cíclicas. }\end{array}$ & $\begin{array}{l}\text { Maclas: múltiples paralelas a } \\
\{001\} \text { y fallas de apilamiento } \\
\{100\} ; \quad \text { maclas cíclicas en } \\
\text { Elba. }\end{array}$ & $\begin{array}{l}\text { Gellens et al. (1982) } \\
\text { Gaines et al. (1997) } \\
\text { Deer et al. (2004) }\end{array}$ \\
\hline $\begin{array}{l}2 V_{y} \rightarrow 55 / 60^{\circ} ; \text { dispersión fuerte } \\
r>V\end{array}$ & $\begin{array}{l}2 \mathrm{~V} \gamma^{7} 55 / 142^{\circ} \text {; dispersión } r>v \\
\text { moderada a fuerte var. cálcica }\end{array}$ & $\begin{array}{l}\text { Deer et al. (2004) } \\
\text { Gaines et al. (1997) } \\
\end{array}$ \\
\hline $\begin{array}{l}\mathrm{T}_{\mathrm{Si}}=0,83-0,85 ; \mathrm{R}^{2+} /\left(\mathrm{R}^{+}+\mathrm{R}^{2+}\right)= \\
0,61 / 0,68 ; \mathrm{DEC}=\text { Ca y } \mathrm{K} .\end{array}$ & $\begin{array}{l}\mathrm{T}_{\mathrm{Si}}=0,77-0,86 ; \mathrm{R}^{2+} /\left(\mathrm{R}^{+}+\mathrm{R}^{2+}\right) \\
=0,53 / 0,84 ; \mathrm{DEC}=\mathrm{Ca}, \mathrm{K}, \mathrm{Na}\end{array}$ & $\begin{array}{lll}\begin{array}{l}\text { Passaglia } \\
(2001)\end{array} & \text { y Sheppard } \\
\end{array}$ \\
\hline
\end{tabular}


TABLA 2. ANÁLISIS QUÍMICOS SELECCIONADOS DE DACHIARDITA.

\begin{tabular}{|l|c|c|c|}
\hline \multicolumn{1}{|c|}{ Óxidos } & Muestra 1 & Muestra 2 & Muestra 3 \\
\hline $\mathrm{SiO}_{2}$ & 68,74 & 67,85 & 68,95 \\
\hline $\mathrm{Al}_{2} \mathrm{O}_{3}$ & 10,68 & 11,49 & 10,54 \\
\hline $\mathrm{CaO}$ & 5,11 & 5,47 & 4,90 \\
\hline $\mathrm{Na}{ }_{2} \mathrm{O}$ & --- & --- & --- \\
\hline $\mathrm{K}_{2} \mathrm{O}$ & 2,47 & 2,20 & 2,60 \\
\hline $\mathrm{MgO}$ & --- & --- & --- \\
\hline$\Sigma$ & 87,00 & 87,01 & 86,99 \\
\hline
\end{tabular}

$\mathrm{O}=48$ a.p.f.u

\begin{tabular}{|l|c|c|c|}
\hline $\mathrm{Si}$ & 20,19 & 19,95 & 20,25 \\
\hline $\mathrm{Al}$ & 3,70 & 3,98 & 3,65 \\
\hline$\Sigma \mathrm{T}$ & 23,89 & 23,93 & 23,90 \\
\hline $\mathrm{Ca}$ & 1,61 & 1,72 & 1,54 \\
\hline $\mathrm{K}$ & 0,93 & 0,82 & 0,98 \\
\hline $\mathrm{Na}$ & -- & --- & --- \\
\hline $\mathrm{T}_{\mathrm{Si}}$ & 0,85 & 0,83 & 0,84 \\
\hline $\mathrm{E} \%$ & $-10,7$ & $-6,7$ & $-10,1$ \\
\hline
\end{tabular}

$E \%=(A I+F e)-(L i+N a+K)-2(M g+C a+S r+B a) /$ $(\mathrm{Li}+\mathrm{Na}+\mathrm{K})+2((\mathrm{Mg}+\mathrm{Ca}+\mathrm{Sr}+\mathrm{Ba}) \times 100, E \%= \pm 10 \%$ según Gottardi y Galli (1985). Tsi= Si/(Si+Al). $\Sigma \mathrm{T}=$ suma de cationes en sitios tetraédricos de la estructura; a.p.f.u.= átomos por fórmula unidad.

valentes: $R^{2+} /\left(R^{+}+R^{+2}\right)=0,61 / 0,68$ concuerda con la citada por Passaglia y Sheppard (2001).

Con los resultados de los análisis químicos de la dachiardita se realizaron los diagramas de la figura 4. Como elementos de comparación se representaron en esa figura, las dachiarditas de la Isla de Elba, Italia (Gottardi y Galli, 1985), Altoona, EE.UU. (Wise and Tschernich, 1978), Onoyama Mine, Japón (Nishido y Otsuka, 1981) y Yellowstone, EE.UU. (Bargar et al., 1987). En la figura 4A realizada en base a los cationes intercambiables de la estructura, se observa que la dachiardita de Esquel, al carecer de $\mathrm{Na}$, forma una serie entre $\mathrm{Ca}$ y K. Hay algunos ejemplos de dachiardita cálcica en Yellowstone (Bargar et al., 1987), cuyos DEC según los autores, son Ca y K; sin embargo no fueron publicados los análisis de esas muestras, por lo que no pudieron ser graficadas en el diagrama $4 \mathrm{~A}$, donde se ubicaron los datos disponibles de otras muestras de Yellowstone. En la figura 4B, que tiene en cuenta a los cationes estructurales, se observa que los análisis de la dachiardita de Esquel están ubicados entre los de la dachiardita de Altoona

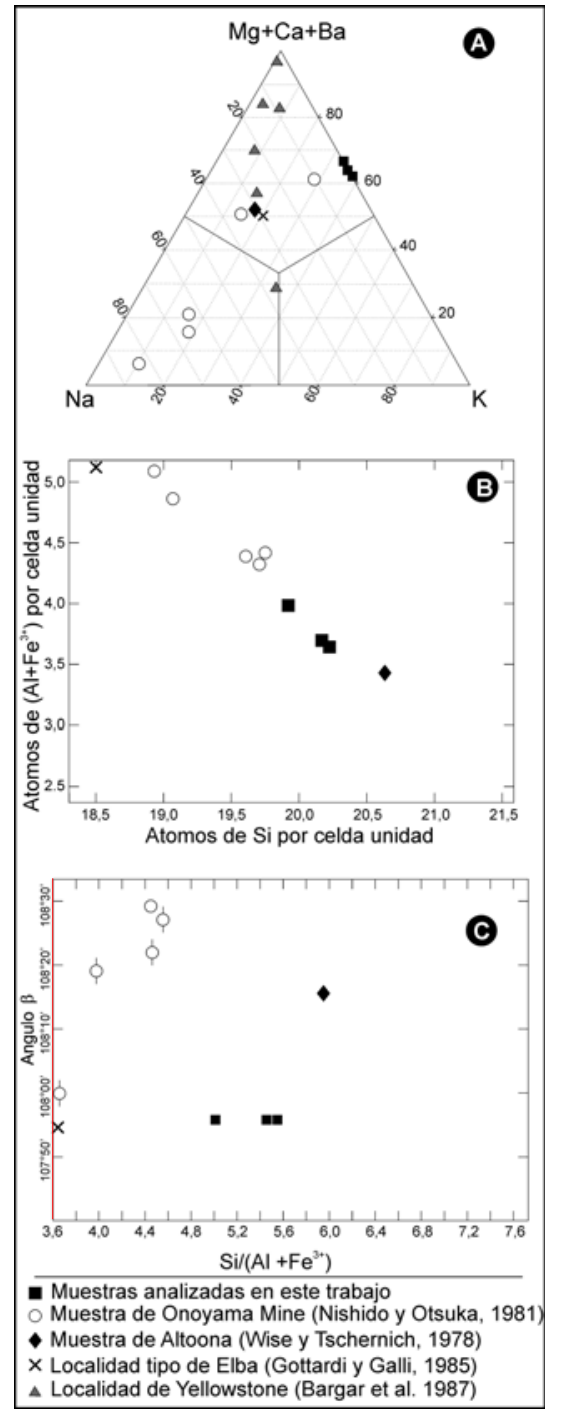

FIG. 4. Representación de los análisis químicos de dachiardita de Esquel a) Diagrama triangular según las relaciones $\mathrm{Na}$ : $\mathrm{Mg}+\mathrm{Ca}+\mathrm{Ba}$ : K (Deer et al., 2004); b) Diagrama de átomos por fórmula unidad (a.p.f.u.) $\mathrm{Al}+\mathrm{Fe}^{+3}$ versus a.p.f.u. Si. Diagrama $\beta$ versus $\mathrm{Si} / \mathrm{Al}+\mathrm{Fe}^{3+}$. Como comparación se representaron algunos análisis de dachiarditas de Elba (Gottardi y Galli, 1985), Altoona, EE.UU. (Wise y Tschernich, 1978), Yellowstone, EE.UU. (Bargar et al., 1987) y Onoyama Mine, Japón (Nishido y Otsuka, 1981).

(Wise y Tschernich, 1978) y los de la de Onoyama Mine (Nishido y Otsuka, 1981).

También se graficó la relación $\mathrm{Si} /(\mathrm{Al}+\mathrm{Fe})=$ $5,01 / 5,55$ versus el ángulo $\beta$ con fines comparativos para proyectarla en el diagrama de la figura $4 \mathrm{C}$ y se observa que está cercana a la dachiardita de Elba. 


\section{DIFRACCIÓN DE RAYOS X Y PARÁMETROS DE LA CELDA UNIDAD}

En la tabla 3 se incluyen los espaciados indexados y las intensidades del diagrama de DRX de dachiardita de Esquel. Éstos coinciden con la ficha 18-467 y 18-467a del 'Mineral Powder Diffraction File Data Book' (Bayliss et al., 1993). El cálculo de los parámetros de la celda se realizó aplicando el método de Holland y Redfern (1997). Estos valores concuerdan con los suministrados para la especie por Armbuster y Gunter (2001).

\section{ZONACIÓN MINERAL METAMÓRFICA}

Las asociaciones metamórficas que acompañan a la dachiardita sugieren la existencia de cuatro etapas de metamorfismo de muy bajo grado (Tabla 4), en orden decreciente de temperatura, de las cuales las etapas II y III corresponden a la facies de ceolita. Durante la etapa I, habría una asociación secundaria en las rocas compuesta principalmente por calcita que se encuentra escasamente en los clastos de la brecha y posteriormente (etapa IV) una asociación de siderita y calcita de segunda generación como cemento de la brecha reemplazando en parte a las ceolitas.

De acuerdo con las relaciones texturales, el orden de depósito inferido para la sucesión minera- lógica secundaria es complicado de establecer para la etapa II, ya que en los clastos de la brecha hay varios minerales que habrían cristalizado simultáneamente, como pectolita, prehnita, adularia, albita, yugawaralita, dachiardita, cuarzo y $\mathrm{S} / \mathrm{C}$ mientras que en esa misma etapa, en el cemento de la brecha y en las venas, cristalizan sólo estos tres últimos minerales (en algún caso albita) y en ocasiones lo hacen alternadamente. En la etapa III, se forman mordenita y cristobalita de baja temperatura seguidas en la etapa IV por siderita y luego calcita de segunda generación, que reemplaza en parte a dachiardita y mordenita en el cemento de la brecha.

\section{DISCUSIÓN Y CONCLUSIONES}

En las escasas localidades en que se encontró en el mundo, la dachiardita se depositó en venas y amígdalas de rocas volcánicas como basaltos, andesitas, riolitas y venas aplopegmatoides. Una asociación con dachiardita, yugawaralita, mordenita, minerales de sílice, siderita y calcita es mencionada por Bargar y Beeson (1984) y Bargar et al. (1987), para Yellowstone, EE.UU. La asociación dachiardita-yugawaralita registra este solo antecedente en el mundo según Passaglia y Sheppard (2001), por lo que la asociación de esas dos ceolitas hallada en Esquel no resulta muy común y sería indicativa de un ambiente geotermal.

La relación $\mathrm{T}_{\mathrm{si}}=\mathrm{Si} /(\mathrm{Si}+\mathrm{Al})=0,83 / 0,85$, se parece a la de Altoona, Washington (Wise y Tschernich, 1978 y Deer et al., 2004).

Según Sheppard y Hay (2001) el $\mathrm{T}_{\mathrm{Si}}$ en las ceolitas tiene una relación directa con el incremento del $\mathrm{H}_{2} \mathrm{O}$ y la $\mathrm{aSiO}_{2}$. El $\mathrm{T}_{\mathrm{si}}$ de la dachiardita de Esquel confirma que es una ceolita que se forma con altas cantidades de Si.

El ángulo $\gamma: \mathrm{C}=43^{\circ}$ se corresponde con el alto contenido en $\mathrm{Ca}$ y con la dispersión fuerte $\mathrm{r}>\mathrm{v}$, según lo sostenido para dachiarditas por Nishido y Otsuka (1981) y también está de acuerdo con los datos suministrados por Bargar et al. (1987), para la dachiardita de Yellowstone. En cambio, el signo óptico negativo es poco común para la dachiardita cálcica según este último autor. Sin embargo, Deer, et al. (2004), manifiestan que el signo óptico y el ángulo de extinción no son confiables para diferenciar entre sí las variedades cálcica y sódica de dachiardita.

No se ha comprobado aún, la influencia que tendría en el signo óptico de la dachiardita, la presencia de $\mathrm{K}$ en lugar de $\mathrm{Na}$.

En cuanto a los minerales asociados a la dachiardita de Esquel, la secuencia paragenética de las ceolitas cálcicas en un sistema geotermal es altamente dependiente del gradiente térmico, de la 
TABLA 3. ESPACIADOS E INTENSIDADES RELATIVAS DEL DIAGRAMA DE DRX DE DACHIARDITA DE ESQUEL.

\begin{tabular}{|c|c|c|c|c|c|}
\hline $\mathbf{d} ?$ & Intensidad & hkl & $\mathbf{d A}$ & Intensidad & hkl \\
\hline 9,78 & 10 & 001 & 2,482 & 20 & 130 \\
\hline 8,90 & 50 & 200 & 2,448 & 10 & 004 \\
\hline 6,90 & 50 & 110 & 2,275 & 5 & -514 \\
\hline 4,97 & 30 & -202 & 2,230 & 5 & 223 \\
\hline 4,45 & 10 & 400 & 2,080 & 10 & -424 \\
\hline 4,23 & 10 & -112 & 1,973 & 20 & -822 \\
\hline 3,820 & 50 & 202 & 1,905 & 10 & 404 \\
\hline 3,636 & 10 & 401 & 1,869 & 75 & 040 \\
\hline 3,490 & 20 & 021 & 1,813 & 30 & 802 \\
\hline 3,460 & 100 & 220 & 1,764 & 35 & -134 \\
\hline 3,325 & 50 & -511 & 1,731 & 20 & -534 \\
\hline 3,190 & 100 & 510 & 1,640 & 10 & 822 \\
\hline 3,020 & 20 & 312 & 1,619 & 20 & 225 \\
\hline 2,865 & 40 & 420 & 1,610 & 10 & 533 \\
\hline 2,550 & 40 & -204 & 1,570 & 10 & -135 \\
\hline
\end{tabular}

\begin{tabular}{|c|c|c|}
\hline Parámetro & valor & sigma \\
\hline $\mathrm{a}_{0}$ & $18,679 \AA$ & 0,033 \\
\hline $\mathrm{b}_{0}$ & $7,488 \AA$ & 0,014 \\
\hline $\mathrm{c}_{0}$ & $10,267 \AA$ & 0,014 \\
\hline$?$ & $107,86^{\circ}$ & 0,207 \\
\hline Vol. celda & $1366,85 \AA$ & 3,280 \\
\hline
\end{tabular}

TABLA 4. PARAGÉNESIS MINERALES SOBRE LA BASE DE TEMPERATURAS DECRECIENTES DESDE LA ETAPA I A IV. ETAPA I: DEPÓSITO DE CARBONATOS; ETAPA II : FACIES DE CEOLITA DE ALTA TEMPERATURA; ETAPA III: FACIES DE CEOLITA DE BAJA TEMPERATURA; ETAPA IV: DEPÓSITO DE CARBONATOS.

\begin{tabular}{|c|c|c|c|c|c|}
\hline Texturas & Clastos de la & brecha & $\mathrm{Cem}$ & ento de la bre & ha y venas \\
\hline Minerales & Etapa I & Etap & oa II & Etapa III & Etapa IV \\
\hline Calcita & --------------------' & & & & --------- \\
\hline Cuarzo & & ---------- & ----------- & & \\
\hline Albita & & ---------- & ---------- & & \\
\hline Prehnita & & ---------- & ---------- & & \\
\hline Pectolita & & ---------- & ---- & & \\
\hline Yugawaralita & & ----------- & & & \\
\hline Adularia & & --------- & & & \\
\hline $\mathrm{S} / \mathrm{C}$ & & ----- & ----------- & & \\
\hline Dachiardita & & ----- & ----------- & & \\
\hline Cristobalita & & & & |------------------- & \\
\hline Mordenita & & & & |------------------- & \\
\hline Siderita & & & & & --o-- \\
\hline
\end{tabular}


razón entre $\mathrm{P}_{\mathrm{H} 2 \mathrm{O}} / \mathrm{P}_{\text {total }} \mathrm{y}$ de la composición de la solución (Giggenbach, 1981).

La yugawaralita asociada, es indicadora de gradiente geotermal alto y presiones muy bajas (Zeng y Liou, 1982) y es muy sensible a la relación $\mathrm{P}_{\mathrm{H} 2 \mathrm{O}} / \mathrm{P}_{\text {total }}$ siendo estable cuando ese valor es alto (entre 0,3 y 1) según Liou et al. (1985) Probablemente esas condiciones posibilitaron que se haya formado junto con dachiardita en los clastos de la brecha, pero nunca en el cemento de la misma, en donde, con menor $\mathrm{P}_{\mathrm{H} 2 \mathrm{O}} / \mathrm{P}_{\text {total }}$ se encuentran solamente las ceolitas dachiardita y mordenita con minerales de sílice y carbonatos, lo que indica menos sensibilidad de estas ceolitas al cambio en la relación $\mathrm{P}_{\mathrm{H} 2 \mathrm{O}} / \mathrm{P}_{\text {total }}$. Las dos ceolitas no se han encontrado en contacto y se presume que la mordenita asociada a cristobalita de baja temperatura y rodeada por carbonatos, sería de más baja temperatura.

La presencia de yugawaralita en la matriz de las rocas acompañando a dachiardita, acota la presión total del proceso metamórfico a menos de $0,5 \mathrm{~kb}$ y las temperaturas a no más de $220^{\circ} \mathrm{C}$ (Zeng y Liou, 1982). Esto se corrobora con la presencia de la asociación yugawaralita + prehnita como reemplazo de los fenocristales primarios en los clastos de la brecha, que reemplazaría, a presiones muy bajas a la asociación laumontita + prehnita en la facies de ceolita (Cho et al. , 1986). Estos autores indican que en esa facies, en ambiente geotermal, se favorece la asociación yugawaralita + prehnita.

Según Frey et al. (1991), el campo de estabilidad de la prehnita a menores presiones que $3 \mathrm{~kb}$ se halla entre los 200 y $280^{\circ} \mathrm{C}$.

La presencia de prehnita, junto con yugawaralita y albita como reemplazo de fenocristales de plagioclasa, y más escasamente en la matriz de rocas afectadas por metamorfismo de muy bajo grado, fue también corroborada unos $100 \mathrm{~km}$ hacia el sudeste de Nahuel Pan, en la localidad de El Molle (Vattuone et al., 1999) en secuencias volcánicas de edad presumiblemente cretácica.

La prehnita de Nahuel Pan, además de hallarse en venas, reemplaza a los fenocristales de plagioclasa y de minerales máficos y está intercrecida indistintamente con yugawaralita o con albita, las que también se encuentran en ese microdominio.

Boles y Coombs (1977) mencionan que la mayor o menor frecuencia de prehnita indica su sensibilidad respecto a la composición de la roca de caja. En casos en que el Ca esté presente en exceso, se favorece la formación de prehnita. Estas mismas condiciones sumadas a la abundancia de Si son requeridas para la formación de dachiardita (Bargar et al., 1987).

En cuanto a la albita, según Liou et al. (1985), en el área geotermal de Onikobe, comienza a cristalizar en la plagioclasa primaria entre los 120 y $180^{\circ} \mathrm{C}$ y a temperatura mayor que $230^{\circ} \mathrm{C}$, los fenocristales primarios de plagioclasa están enteramente reemplazados por albita, cosa que no ocurre en nuestro caso. Según Boles (1982), las fracturas de las rocas favorecen la albitización, especialmente las que son importantes en extensión. También Marrett (1996), indica que las fracturas importantes en extensión constituyen un medio que controla la permeabilidad total. Como se indicó en el capítulo de contexto geológico, las brechas estudiadas en este trabajo corresponden a brechas de falla que forman parte de estructuras regionales de rumbo noerest-sureste.

La adularia es relativamente abundante en los clastos, especialmente cercana a las venas y es indicadora de la presencia de potasio en los fluidos, que podría ser aportado por soluciones, que habrían reaccionado con la andesina primaria y depositado feldespato potásico secundario. Esto fue estudiado entre otros, por Zeng et al. (1998), en un trabajo experimental.

Browne y Ellis (1970) identificaron en un área geotermal de Nueva Zelanda (Ohaki-Broadlands) tres caminos para la formación de adularia: 1) reemplazo de plagioclasa primaria; 2) introducción de significante $\mathrm{K}$; y 3 ) incremento en $\mathrm{pH}$ debido a ebullición del $\mathrm{H}_{2} \mathrm{O}$ con precipitación de feldespato potásico y $\mathrm{CaCO}_{3}$. En el presente caso, se consideró factibles las dos primeras hipótesis, pero no se tiene elementos de juicio que prueben que existió la tercera posibilidad, aunque es probable que así fuera, de acuerdo a los minerales formados, cuarzo, feldespato potásico y $\mathrm{CaCO}_{3}$.

Los procesos secundarios comenzaron, casi con seguridad, con posterioridad al brechamiento de la roca. En la figura 5, se proponen las reacciones que habrían tenido lugar a partir de la destrucción de la plagioclasa primaria que condujo a la precipitación de los principales minerales secundarios y se esquematiza la variación de los factores que afectan a dichas reacciones.

A partir de la destrucción de la plagioclasa 


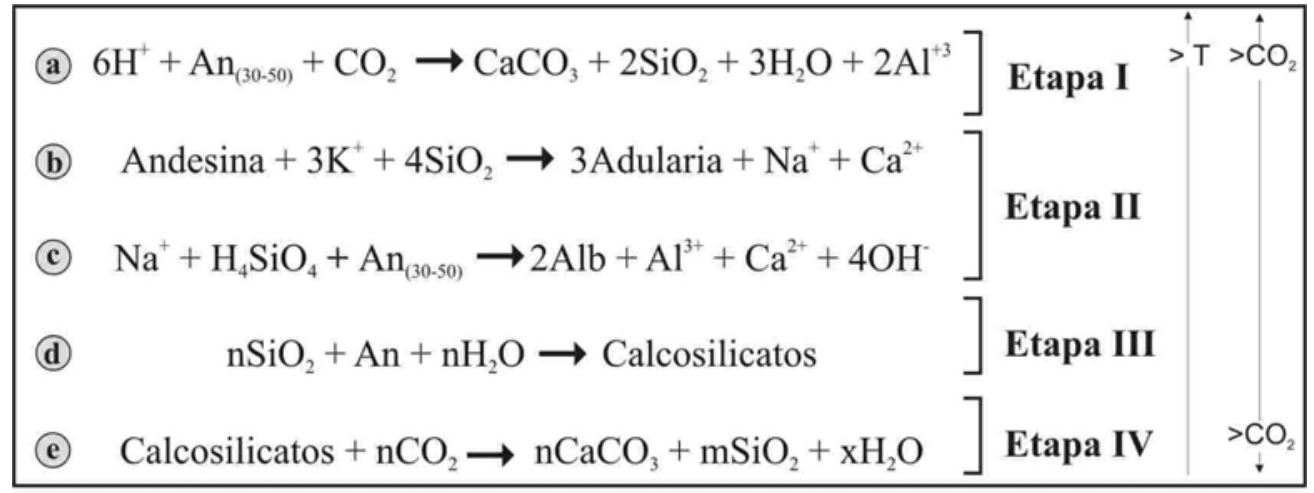

FIG. 5. Esquema de las principales reacciones involucradas en la formación de los minerales secundarios; a) modificada de Giggenbach (1981); b) Martins e Hippert (1987) c) Boles (1982) d) Coombs et al. (1959).

primaria en ambiente ácido, el $\mathrm{pH}$ se va haciendo alcalino y se habría mantenido relativamente constante según nuestra hipótesis a lo largo de todas las etapas lo que posibilita la formación de carbonatos y calcosilicatos. En una primera, la $\mathrm{X}_{\mathrm{CO} 2}$ habría sido un factor importante, que contribuyó a la destrucción de la plagioclasa cálcica. La calcita que se observa reemplazando a la plagioclasa se habría formado por el calcio perdido por la misma al reaccionar con el $\mathrm{CO}_{2}$ del fluido en esta primera etapa.

En la segunda etapa, en los clastos de la brecha, se forman albita y adularia. La presencia de esta última restringida a las cercanías de las venas, sugiere que el $\mathrm{K}$ habría sido introducido en un ambiente acuoso. Una baja $\mathrm{X}_{\mathrm{CO} 2}$ permitió la formación de los calcosilicatos (pectolita, prehnita, yugawaralita y escasa dachiardita).

Al mismo tiempo, en el cemento de la brecha y en venas, la dachiardita registra gran abundancia ya que encontró allí un ambiente de formación propicio, con alta $a_{\mathrm{SiO}_{2}}$ evidenciado por la presencia de minerales de sílice y alta concentración de $\mathrm{Ca}$ en los fluidos termales, lo cual favorece la formación de esta ceolita tal como lo sostienen Bargar et al. (1987) para la dachiardita de Yellowstone. Dachiardita y mordenita, son consideradas las ceolitas más silíceas, según Chippera y Apps (2001).

En consecuencia, las temperaturas a las que se forma la asociación de yugawaralita, adularia, albita con plagioclasa relicta e interestratificados de S/C, indicarían para la formación de dachiardita, valores entre 200 y $220^{\circ} \mathrm{C}$ a presiones muy bajas. Esto está de acuerdo con las temperaturas máximas a las que cristalizaría la dachiardita de Yellowstone determinadas en base a técnicas de inclusiones fluidas en el cuarzo acompañante (Bargar et al., 1987).

La asociación mordenita - cristobalita se habría formado con un leve descenso de la temperatura; según Passaglia y Sheppard (2001), en el campo geotermal de Wairakei, la mordenita se forma a temperaturas entre 150 y $230^{\circ} \mathrm{C}$, lo que concuerda

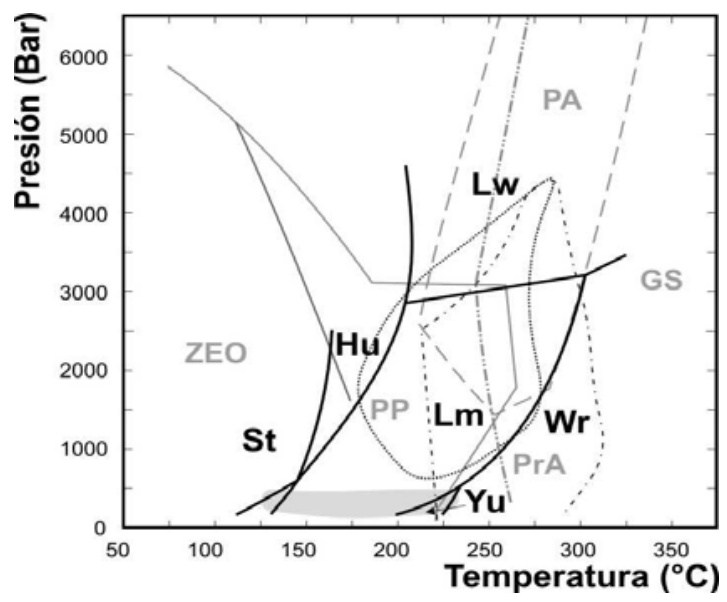

FIG. 6. Red petrogenética P-T para el metamorfismo de muy bajo grado (VLGM) en metabasitas (Frey et al., 1991; Frey y Robinson, 1999). ZEO: facies ceolita; PP: facies de prehnita pumpellyita; PrA: facies de prehnita-actinolita; PA: facies de pumpellyita-actinolita y GS: facies de esquistos verdes. Las curvas de equilibrio de las ceolitas (línea gruesa) están tomadas de Cho et al. (1987) y de Zeng y Liou (1982). St: estilbita; Hu: heulandita; Lm: laumontita; Yu: yugawaralita; Wr: wairakita y Lw: lawsonita. El sector sombreado corresponde a las condiciones $\mathrm{P}$-T inferidas para los procesos metamórficos de este trabajo. 
con el campo de estabilidad en forma metaestable de la cristobalita de baja temperatura $(\beta)$ que suele encontrarse a temperaturas entre $120^{\circ} \mathrm{C}$ y $272^{\circ} \mathrm{C}$ (Deer et al., 2004).

Posteriormente, los fluidos enriquecidos en calcio favorecidos por el aumento de la $\mathrm{X}_{\mathrm{CO} 2}$ en las zonas de fractura depositaron carbonatos de segunda generación, siderita y luego calcita en las venas, a temperaturas menores. Bargar y Beeson (1984), para paragénesis similares, postulan para la siderita, temperaturas menores a $120^{\circ} \mathrm{C}$ que podrían llegar hasta un mínimo de $80^{\circ} \mathrm{C}$ y sostienen, basándose en algunos ejemplos de localidades de
Nueva Zelanda que ese mineral se formaría por lo común por debajo de $130^{\circ} \mathrm{C}$.

En la figura 6 se ubica el campo de presión y temperatura a las que se habrían formado las asociaciones con dachiardita.

Por otra parte, la ausencia de Na y la presencia de $\mathrm{K}$, en la dachiardita de Esquel, implica una composición algo particular, conocida de muy pocos lugares en el mundo (sólo citada para Yellowstone por Bargar et al., 1987) y poco común en las ceolitas de los Andes Nordpatagónicos. Esto se relacionaría, quizás, con la presencia de adularia, de la cual podría haberse formado la dachiardita.

\section{AGRADECIMIENTOS}

Los autores desean agradecer a la Universidad de Buenos Aires y al CONICET con cuyo apoyo, por medio de los proyectos Ubacyt X238 y PIP No.
2244 , se realizaron las investigaciones. Se agradece a los señores árbitros las sugerencias realizadas.

\section{REFERENCIAS}

Armbruster, T.; Gunter, M. 2001. Crystal Structures of Natural Zeolites. In Natural Zeolites: Reviews in Mineralogy y Geochemistry (Bish, D.; Ming, D.; editores). Mineralogical Society of America 45: 1-67. Washington, DC. EE.UU.

Bargar, K.E.; Beeson, M. 1984. Hydrothermal alteration in Research Drill Hole Y-6, Upper Firehole river, Yellowstone National Park, Wyoming. United States Geological Survey, Professional Paper 1054B: B1B22.

Bargar, K.; Erd, R.; Keith, T.; Beeson, M. 1987. Dachiardite from Yellowstone National Park, Wyoming. Canadian Mineralogist 25: 475-483.

Bayliss, P.; Erd, D.C.; Mross, M.E.; Sabina, A.P.; Smith, D.K. 1993. Mineral Powder Diffraction File. Compiled for the Joint Committee on Powder Diffraction Standard. Mrose, M.E. Editors: 1396 p. Pasadena, USA.

Bermann, H. 1925. Notes on dachiardite. American Mineralogist 10: 421-428.

Boles, J. 1982. Active albitization of plagioclase, Gulf Coast, Tertiary. American Journal of Science 282: 165-180.

Boles, J.; Coombs, D. 1977. Zeolite facies alteration of sandstones in the Southland syncline, New Zealand. American Journal of Science 277: 982-1012.

Bonardi, M.; Roberts, A.; Sabina, A.; Chao, G. 1981. Sodium-rich dachiardite from the Francon Quarry,
Montreal Island, Quebec. Canadian Mineralogist 19: 285-289.

Browne, P.R.; Ellis, A.J. 1970. The Ohaki-Broadlands hydrothermal area, New Zealand: mineralogy and related geochemistry. American Journal of Science 269: 97-131.

Coombs, D.S. 1997. Recommended nomenclature for zeolite minerals: report of the Subcommittee on zeolites of the International Mineralogical Association, Comission on new minerals and mineral names. The Canadian Mineralogist: 35: 1571-1606.

Coombs, D.S.; Ellis, A.; Fyfe, W.; Taylor, A. 1959. The zeolita facies with comments on the interpretation of hidrotermal syntheses. Geochimica et Cosmochimica Acta 17: 53-107.

Chippera, S.; Apps, J. 2001. En Natural Zeolites: Reviews in Mineralogy y Geochemistry, Vol. 45 (Bisch, D.; Ming, D.; editors). Mineralogical Society of America: 117-161. Washington, DC, USA.

Cho, M.; Liou, J.; Maruyama, S. 1986. Transition from the zeolite to prehnite-pumpellyite facies in the Karmutsen metabasites, Vancouver Island, British Columbia. Journal of Petrology 27 (2): 467-494.

Cho, M.; Maruyama, S.; Liou J. 1987. An experimental investigation of heulandite-laumontite equilibrium at 1000 to 2000 bar $\mathrm{P}_{\text {fluid }}$. Contribution to Mineralogy and Petrology 97: 43-50. 
de la Cruz, R.; Suárez, M.; Belmar, M.; Quiroz, D.; Bell, M. 2003. Geología del área Coyhaique-Balmaceda, Región Aisén del General Carlos Ibáñez del Campo. Servicio Nacional de Geología y Minería, Carta Geológica de Chile, Serie Geología Básica (80): 40 p.

Deer, W.; Howie, R.; Zussman, J.; Wise, W. 2004. Rock Forming Minerals. Vol. 4B. Framework Silicates. The Geological Society, 2 Edición: 982 p. London.

Depine, G.; Gargiulo F.; Leal P.; Scaricabarozzi N.; Spagnuolo C.; Vattuone, M.E. 2003. Paragénesis de ceolitas en rocas volcánicas de la Cordillera Patagónica Septentrional, Villa La Angostura, Neuquén, República Argentina. In Congreso Geológico Chileno, No. 10, Actas:10 p. Concepción.

Folguera, A.; lanizzotto, N. 2004. The Lagos Fontana and La Plata fold and thrust belt: long lived orogenesis at the edge of western Patagonia. Journal of South American Earth Science 16: 541-566.

Frey, M.; De Capitani, C.; Liou, J. 1991. ANew Petrogenetic Grid for Low-Grade Metabasites. Journal of Metamorphic Geology 9: 497-509.

Frey, M.; Robinson, D. 1999. Low Grade Metamorphism. Blackwell Science Ltd.: 313 p. London, Gran Bretaña.

Gaines, R.; Skinner, H.; Foord, E.; Mason, B.; Rosenzweig, A.; King, A. 1997. Dana's New Mineralogy. John Wiley y Sons Inc.: 1819 p. New York, EE.UU.

Gellens, L.; Price, G.; Smith, J. 1982. The structural relation between svetlozarite and dachiardite. Mineralogical Magazine 45: 157-161.

Giggenbach, W. 1981. Geothermal mineral equilibria. Geochimica et Cosmochimica Acta 45: 393-410.

Gottardi, G.; Galli, E. 1985. Natural zeolites. SpringerVerlag: 409 p. Berlin.

Haller, M.; Lapido, O. 1982. The Jurassic-Cretaceous volcanism in the Septentrional Patagonian Andes. Earth Science Reviews 18: 395-410.

Holland, T.; Redfern, S. 1997. Unit cell refinement from powder diffraction data: the use of regression diagnostics. Mineralogical Magazine 61: 65-77.

Ianizzotto, N.; Folguera, A.; Leal, P.; Yaffa, D. 2003. Ambiente de acumulación de las secuencias volcaniclásticas neocomianas y evolución tectónica en la zona del Lago La Plata $\left(45^{\circ} \mathrm{S}\right)$. In Congreso Geológico Chileno, No. 10, Actas 1: 10 p. Concepción.

Lagorio, S.; Massaferro G.; Vattuone, M.E.; Latorre, C. 2001. Mineralogía y metamorfismo de vulcanitas de Aluminé. Revista de la Asociación Geológica Argentina 56 (2): 211-220.

Latorre, C.; Vattuone, M.; Massaferro, G.; Lagorio, S.. 1990. Analcima, Thomsonita, Laumontita y Natrolita en basaltos de Rucachoroi: mineralogía y condiciones de formación. Revista de Geólogos Economistas, Publicación especial: 18-26. Buenos Aires.

Latorre C.; Vattuone, M.; Leal, P. 2000. Hallazgo de barrerita en amígdalas de metabasaltos, noroeste de Chubut, Patagonia Argentina. In Simposio Internacional de Metamorfismo Andino. In Congreso
Geológico Chileno, No. 9, Actas 2: 515-519. Puerto Varas.

Liou, J.; Seki, Y.; Guillemette, R.; Sakai, H. 1985. Composition and parageneses of secondary minerals in the Onikobe geothermal system, Japan. Chemical Geology 49: 1-20.

Lizuaín, A. 1999. El Jurásico y Cretácico de la Patagonia y Antártida. Estratigrafía y evolución geológica del Jurásico y Cretácico de la Cordillera Patagónica Septentrional. Geología Argentina (Caminos, R.; editor). Instituto de Geología y Recursos Minerales, Anales 29, 17: 433-556. Buenos Aires.

Martins Hippert, J.F. 1987. Texturas indicativas de metassomatismo potássico nos Augen/Gneisses de Niteroi, RJ. Revista Brasileira de Geociencias 17 (3): 253-262.

Marrett, R. 1996. Aggregate properties of facture populations. Journal of Structural Geology 18: 169178.

Nishido, H.; Otsuka, R. 1981. Chemical composition and physical properties of dachiardite group zeolites. Mineralogical Journal, The Mineralogical Society of Japan 10 (8): 371-384.

Pankhurst, R.J.; Leat, P.; Sruoga, P.; Rapela, C.; Márquez, M.; Storey, B.; Riley, T. 1998. The Chon Aike province of Patagonia and related rocks in West Antarctica: a silicic large igneous province. Journal of Volcanology and Geothermal Research 81: 113-136.

Passaglia, E.; Sheppard, R. 2001. The crystal chemistry of zeolites. In Natural Zeolites. Reviews in Mineralogy y Geochemistry (Bisch, D.; Ming, D.; editores). Mineralogical Society of America: 69-115. Washington, DC., EE.UU.

Pesce, A.H. 1978. Estratigrafía de la Cordillera Patagónica entre los $43^{\circ} 30^{\prime}$ y $44^{\circ}$ de latitud sur. In Congreso Geológico Argentino, No. 7, Actas 1: 257-270. Neuquén.

Ramos, V.A. 1976. Estratigrafía de los Lagos La Plata y Fontana, Provincia del Chubut. In Congreso Geológico Chileno, No. 1, Actas 1: A43-A64. Santiago.

Ramos, V. 1978. El vulcanismo del Cretácico Inferior de la Cordillera Patagónica. In Congreso Geológico Argentino, No. 7, Actas 1: 423-435. Neuquén.

Ramos, V. 1981. Descripción Geológica de la Hoja 47abLago Fontana, Provincia de Chubut. Servicio Geológico Nacional, Boletín 183: 183 p. Buenos Aires.

Secretaría de Minería, Dirección Nacional del Servicio Geológico. 1995. Mapa Geológico de la Provincia del Chubut. Dirección Nacional del Servicio Geológico, Secretaría de Minería, escala 1:750.000.

Sheppard, R.; Hay, R. 2001. Occurrence of zeolites in sedimentary rocks: an overview. In Natural Zeolites: Reviews in Mineralogy y Geochemistry (Bisch, D.; Ming, D.; editores). Mineralogical Society of America 45: 217-234. Washington, DC. EE.UU.

Tourn, S.; Vattuone, M.E. 2002. Cobre nativo y cuprita en una paragénesis ceolítica en amígdalas de lavas 
basálticas, Chapelco, Provincia de Neuquén. In Mineralogía y Metalogenia 2002 (Brodtkorb, M.; Kouharsky, V.X.; editores). Asociación Mineralógica Argentina (AMA) 1: 425-432. Buenos Aires.

Vattuone, M.; Latorre, C. 1990. Low grade metamorphism in granitoids and volcanic rocks of Cordillera Neuquina, Argentina. Journal of South America Earth Sciences 3 (4): 247-252.

Vattuone, M.E.; Latorre, C. 1996a. Yugawaralita de Lago Meliquina, Neuquén. In Reunión de Mineralogía y Metalogenia, No. 3. Instituto de Recursos Minerales (INREMI), Universidad Nacional de La Plata (UNLP), Publicación (5): 251-256. La Plata.

Vattuone, M.E.; Latorre, C. 1996b. Metamorfismo de muy bajo grado en rocas volcánicas de la Formación Ventana, San Martín de los Andes, Neuquén, Argentina. Revista Geológica de Chile 23: 187-200.

Vattuone, M.E.; Latorre, C.; Viviani, R.; Borbolla, M. 1996. Mineralogía de ceolitas cálcicas y filosilicatos máficos que caracterizan el metamorfismo hidrotermal de las volcanitas paleógenas desde Lago Hermoso a Río Traful, Neuquén. Revista de la Asociación Geológica Argentina 51: 235-247.

Vattuone, M.E.; Latorre, C.; Leal, P.; Martínez, A.; Viviani, R. 1999. Calcosilicatos y filosilicatos de facies ceolita y prehnita-pumpellyita en Pío Proto, Neuquén, Patagonia Argentina. Boletín de la Sociedad Española de Mineralogía 22: 185-197.

Vattuone. M.E.; Latorre, C. 1999. Ceolitas cálcicas en vulcanitas del Cretácico Superior. Su génesis en un paleosistema geotermal. El Molle, Chubut, República Argentina. Boletín del Primer Simposio sobre el Cretácico de América del Sur, Serra Negra, Brasil. Publicación trienal 1: 213-218.

Vattuone, M.E.; Latorre, C.; Leal, P. 2001a. Procesos de formación de paragénesis ceolíticas en el metamorfis- mo de muy bajo grado de las volcanitas paleógenas al sur de Confluencia, Neuquén, Argentina. Revista Geológica de Chile 28 (2): 3-22.

Vattuone, M.E.; Latorre, C.; Leal, P. 2001b. Barrerita en metavolcanitas de Confluencia, Neuquén, Patagonia Argentina. Boletín de la Sociedad Española de Mineralogía 24: 23-32.

Vattuone, M.E.; Latorre, C. 2002. Na-Mg offretite from Futalaufquén, Patagonian Andes, Argentina. Zeolite '02. Occurrence, Properties and Utilization of Natural Zeolites. International Conference, No. 6. Thessaloniki, Abstracts 1: 382-383. Greece.

Vattuone, M.E.; Latorre, C. 2004. Edades K/Ar al este del Cerro Nahuel Pan, Chubut. Implicancias en la correlación del Grupo Divisadero y del Choiyoi en el área. Revista de la Asociación Geológica Argentina 59 (3): 510-513.

Vezzalini, G. 1984. A refinement of Elba dachiardite: opposite acentric domains simulating a centric structure. Zeitschrift für Kristallographie 166: 63-71.

Wise, W.; Tschernich, R. 1978. Dachiardite-bearing zeolite assemblages in the Pacific Northwest. In Natural Zeolites: occurrence, properties, use (Sand, L.; Mumpton, F.; editors). Pergamon Press, Serie E: 105-111. New York, EE.UU.

Yoshimura,T.; Wakabayasi, S. 1977. Na dachiardite and associated high-silica zeolites form Tsugawa, northeast Japan. Science Reports Niigata University, Serie E, Geological Mineralogist 4: 49-64.

Zeng,Y.; Ai, R.; Ai, Y. 1998. Experimental study on the flow-trough interaction between andesine and alkalichloride hydrothermal solutions. News Jahrbuch fur Mineralogie Monatshefte 5: 193-207.

Zeng, Y.; Liou, J. 1982. Experimental investigation of yugawaralite-wairakite, equilibrium. American Mineralogist 67: 937-943. 


\section{APÉNDICE 1}

\section{METODOLOGIA}

La metodología empleada consistió en el reconocimiento geológico del área y el muestreo de los afloramientos de las secuencias volcánicas en la localidad que se ubica en la figura 1. Se extrajeron 30 muestras para estudiar la petrografía y la mineralogía. En el laboratorio se procedió al estudio óptico de la dachiardita bajo el microscopio petrográfico y electrónico de barrido ambiental (ESEM) marca Phillips Electroscan Modelo 2010. Se analizó por difracción de rayos $X(D R X)$ mediante el método Debye Scherrer realizado con un difractómetro Siemens, modelo D5000. La longitud de onda utilizada para el cálculo fue $\mathrm{CuK}_{\alpha 1=} 1,54056 \AA$, con filtro de $\mathrm{Ni}$, escaneado a una velocidad de $1,2^{\circ} 2 \theta /$ minuto, desde $2 \theta=5-60^{\circ}$. Los parámetros de la celda unidad fueron obtenidos por refinamiento de los datos de DRX usando el programa de computación de Holland y Redfern (1997). Las ceolitas dachiardita y yugawaralita fueron analizadas químicamente en los laboratorios del Centro de Investigaciones Técnicas de la Fuerzas Armadas (CITEFA), mediante espectrómetro de energía dispersiva (EDS), con un microscopio electrónico de barrido (SEM) Phillips 9100. Los patrones utilizados fueron elementos metálicos ( $\mathrm{Si}, \mathrm{Al}, \mathrm{Ca}, \mathrm{Na}, \mathrm{K}, \mathrm{Mg}$ y Ba). Los análisis se realizaron a $20 \mathrm{kv}$, corrección convencional ZAF y TKOFF $=25^{\circ}$. El haz de electrones que se usó es de 2 micrones de diámetro y el tiempo de medición fue de 10 segundos. El error promedio de los datos de EDS para cada elemento es de 2,6\% en peso. Los cationes $\mathrm{Na}, \mathrm{Mg}$ y Ba están por debajo del límite de detección que es menor al 1\%. Los tres análisis de la Tabla 2 que fueron seleccionados de un total de 10, representan el promedio de un área elegida en cada cristal, que abarcó unos $10 \mu \mathrm{m}$. En cada área se analizaron 5 puntos.

Como la cantidad de muestra no fue suficiente para efectuar termogravimetría, se estimó el contenido de $\mathrm{H}_{2} \mathrm{O}$ tomando las cifras teóricas de Deer et al. (2004).

Los minerales acompañantes de la dachiardita fueron determinados ópticamente y además, por DRX. La cristobalita fue identificada como cristobalita $\beta$ y la albita como albita de baja temperatura. La adularia fue determinada además por su maclado. Los individuos de S/C constituyen interestratificados no regulares y fueron determinados por DRX y ópticamente.

En el caso de la yugawaralita se realizaron análisis químicos por EDS (a.p.f.u en base a 32 O) y se obtuvo Si:11,78; Al: 4,18; Ca: 2,17. E\%=-3.53. 